\title{
Anterior Communicating Artery Aneurysm with a Peculiar Angiographic Appearance: A Case Study
}

\author{
Masato OTAWA, ${ }^{1}$ Takashi IZUMI, ${ }^{1}$ Masahiro NisHIHORI, ${ }^{1}$ Tetsuya TsuKADA, ${ }^{1}$ \\ Ryosuke OsHIMA, ${ }^{1}$ Tomomi KAWAGUCHI, ${ }^{1}$ Shunsaku GOTO, ${ }^{1}$ \\ Mizuka IKEZAWA, ${ }^{1}$ Asuka Elisabeth KROPP, ${ }^{1}$ and Toshihiko WAKABAYASHI ${ }^{1}$ \\ ${ }^{1}$ Department of Neurosurgery, Nagoya University Graduate School of Medicine, Nagoya, \\ Aichi, Japan
}

\begin{abstract}
We describe a case of pulsatile anterior communicating artery aneurysm (A-com AN) with a peculiar angiographic appearance. An 81-year-old man was referred to the department of neurosurgery for a large A-com AN artery aneurysm, which was detected incidentally. The patient hoped not to be treated but to be followed up. After 4 months, magnetic resonance imaging (MRI) revealed the presence of a cerebral edema and hematoma around the aneurysm, and partial thrombus in the upper wall of the aneurysm was suspected. Based on these findings, the patient underwent an immediate coil embolization a day after admission. However, the portion of the neck remnant increased in size after the first procedure. Therefore, 8 months after the initial procedure, he was treated with stent coil embolization. Contrary to the first procedure, angiographic evaluation revealed an active pulsating aneurysm. Moreover, MRI revealed the presence of a partial thrombus in the upper neck segment of the aneurysm, with an intensity that changed over time. The patient underwent cautious treatment and was discharged without any symptoms. This is the first case study to reveal an A-com AN with active pulsation and the relationship between the pulsatile portion of the aneurysm and thrombosed portion by MRI.
\end{abstract}

Keywords: angiographic appearance, partial thrombosed aneurysm, pulsatile aneurysm

\section{Introduction}

We experienced a case of pulsatile intracranial aneurysm with a peculiar angiographic appearance. A head magnetic resonance imaging (MRI) revealed a partial thrombus above the enhanced pulsatile region. To the best of our knowledge, this is the first case to report a pulsatile aneurysm, and to reveal the relationship between an aneurysm's partial thrombus and pulsation by MRI.

\section{Case Report}

An 81-year-old man was referred to our hospital due to a large anterior communicating artery aneurysm (A-com AN) detected incidentally. The size

Received February 6, 2020; Accepted May 11, 2020

Copyright $\subseteq 2021$ by The Japan Neurosurgical Society This work is licensed under a Creative Commons AttributionNonCommercial-NoDerivatives International License. was about $14 \mathrm{~mm}$. However, the presence of a partial thrombus was not confirmed, which was attributed to poor visualization of the area. Specifically, factors such as the large thickness of the imported MRI image and lack of sagittal images were considered crucial. The patient went to the hospital for a follow-up, not treatment. After 4 months, he underwent a follow-up MRI, which revealed cerebral edema and hematoma around the aneurysm. A partial thrombus in the upper wall of the aneurysm was suspected (Fig. 1). We were unable to determine whether the hematoma was within the intra-aneurysmal wall or whether an intracerebral hemorrhage was present. The patient was asymptomatic. However, he was admitted in emergency and underwent transarterial embolization (TAE) the following day.

The patient was treated with a double catheter technique. A perforator arising from the aneurysmal neck was visualized, and coil embolization was performed with caution to preserve the perforator. A 392-cm-long coil was placed in the aneurysm 

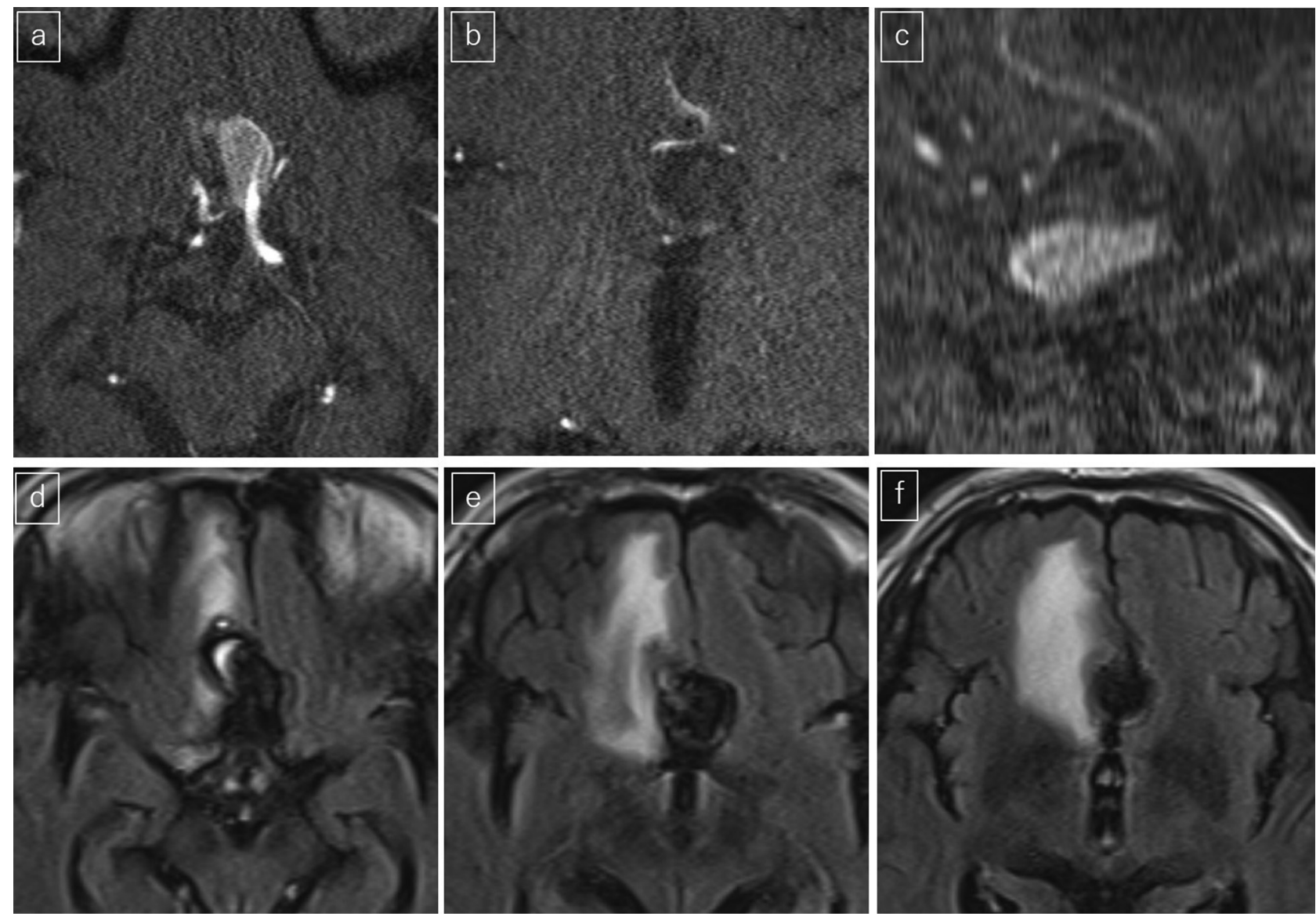

Fig. 1 Four months after the first visit, a follow-up MRI demonstrated a cerebral edema and hematoma around the aneurysm. A partial thrombus in the aneurysmal upper wall was suspected. (a-c) Follow-up time-of-flight magnetic resonance angiography image 4 months after the first visit. (a and b) axial view, (c) sagittal view, (d-f) follow-up MRI, FLAIR image axial view 4 months after the first visit. A sagittal view was not taken, therefore, findings that we suspected partial thrombus part was not shown. A cerebral edema and hematoma around the aneurysm were demonstrated. We were unable to clarify whether the hematoma was in the intra-aneurysmal wall or due to a cerebral hemorrhage. FLAIR: fluid-attenuated inversion recovery, MRI: magnetic resonance imaging.

(Fig. 2). As a result, the patient had a large neck remnant. He was discharged with no symptoms. We decided that if the part of the neck remnant increased in size, the patient would then undergo a TAE with a stent. Follow-up MRI was performed. The patient remained free from neurological symptoms since the first TAE. However, 8 months after the initial TAE, an increase in the neck remnant part of the aneurysm was observed. In addition, T1-weighted image showed the presence of a partial thrombus in the upper neck segment of the aneurysm, with intensity that had changed over time (Fig. 3). Based on these findings, a second TAE was conducted 8 months after the first procedure.

We performed TAE with the stent-assisted technique. Under local anesthesia, an 8-Fr guiding catheter (Slim Guide; Medikit, Tokyo, Japan) was advanced coaxially into the left internal carotid artery using a 6-Fr guiding catheter (Cerulean DD6; Medikit, Tokyo, Japan). Then, a three-dimensional rotational angiogram (3D-RA) was performed. This revealed a pulsatile neck segment of the aneurysm (Video). For the first time, we were able to visualize the pulsatile aneurysm. Notably, at the time of 3D-RA during the first TAE, the pulsatile aneurysm was not observed (Video, available online). We cautiously advanced an Excelsior SL-10 microcatheter (Boston Scientific Corp., Fremont, CA, USA) into the right anterior cerebral artery to place an aneurysmal stent. Another Excelsior SL-10 microcatheter was advanced into the aneurysm and was placed in a total coil length of $166 \mathrm{~cm}$ (Fig. 4). When the coil mass was extended to the anterior communicating artery, a Neuroform Atlas stent (Stryker Neurovascular, Fremont, CA, USA) measuring $4.5 \times 30 \mathrm{~mm}$ was placed from the 

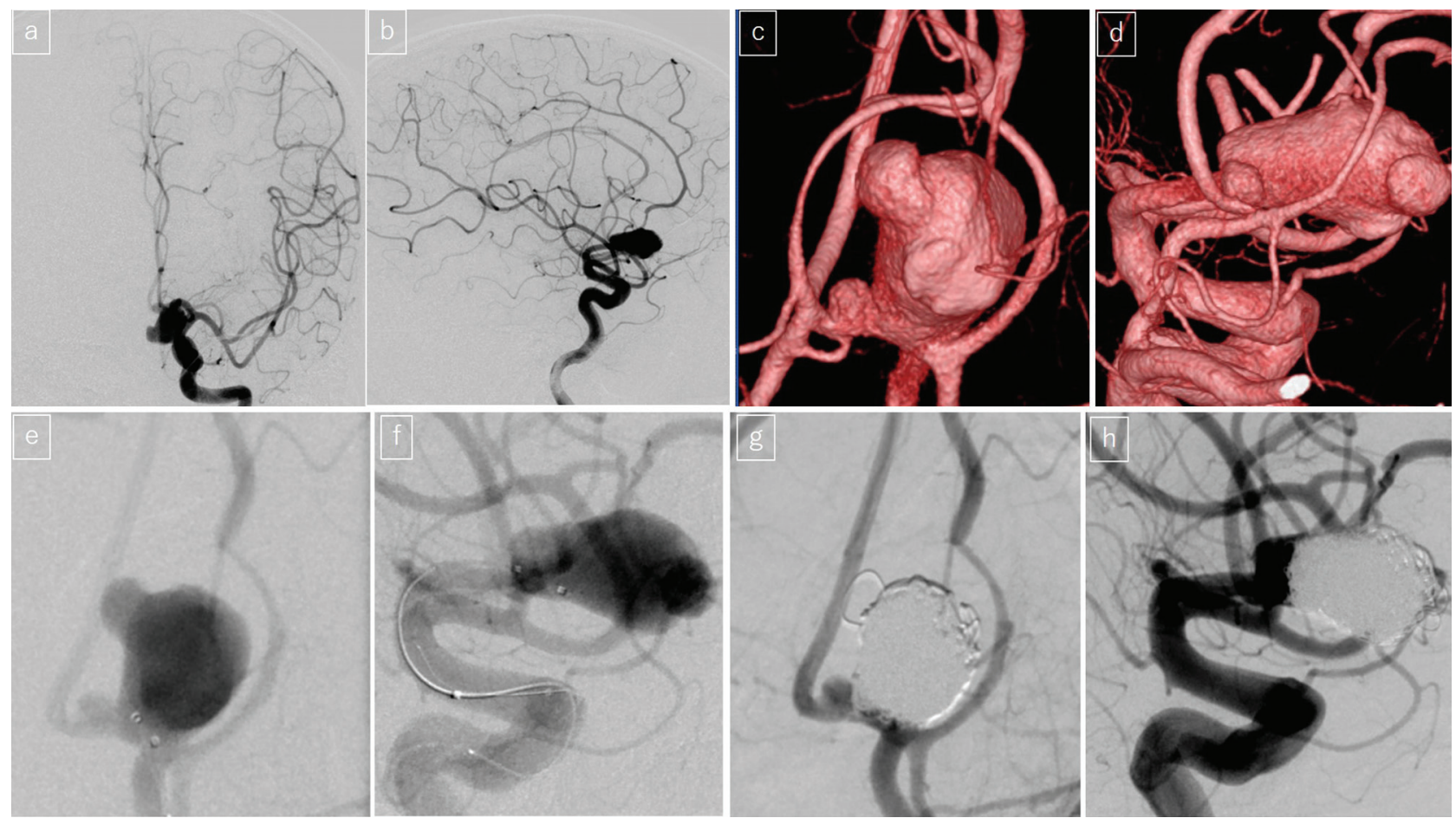

Fig. 2 We performed a TAE with double catheter technique for the A-com AN. A perforator arose from the aneurysmal neck and then, coil embolization was performed carefully not to compromise its blood flow. As a result, a slightly large neck part remained. (a and b) Pre-treatment left internal carotid angiogram. (a) anteroposterior (AP) view, (b) lateral view. (c and d) 3D-RA volume-rendering image. Enlarged working angle view. (e and f) Working angle view. (g and h) Post-procedural image. A large neck remnant was demonstrated because a perforator was detected around the neck by a 3D-RA image. 3D-RA: three-dimensional rotational angiography, A-com AN: anterior communicating artery aneurysm, TAE: transarterial embolization.

right A2 to the left A1 segment. A perforator arising from the aneurysmal neck was preserved similar to the first TAE.

The patient was asymptomatic and was discharged after a few days.

\section{Discussion}

In the present report, we described a case of pulsatile aneurysm with a peculiar angiographic appearance. To the best of our knowledge, only two similar cases have been reported in the literature to date. ${ }^{1,2)} \mathrm{Xu}$ et al. have reported a case of a ruptured blister aneurysm in the supra-clinoid portion of the internal carotid artery, which increased in size within a short time. The authors have observed active pulsation of a blister aneurysm based on angiographic examination. ${ }^{1)}$ During direct surgery, a blister aneurysm with a fragile, thin and pinkish membrane was found. Moreover, Malek et al. have reported a case of a ruptured middle cerebral artery aneurysm, which was pulsating when observed on angiogram. ${ }^{2}$ They have postulated that elevated intracranial pressure caused by aneurysm rupture and high interstitial pressure attributed to the hematoma surrounding the aneurysm, decreased the transmural pressure gradient, and led to a partial collapse of the compliant aneurysm wall. As a result, the aneurysm showed a pulsating appearance.

In the present case, the A-com AN was a partially thrombosed aneurysm, and the thrombus segment was above the pulsatile part by the MRI images. However, these were not used in the two cases previously reported in the literature. Therefore, whether the aneurysm included a partial thrombus was not validated.1)

In the present case, we hypothesized that the pulsation may be attributed to two mechanisms. First, the entire aneurysmal wall, including the partial thrombus, was pulsating. Specifically, the angiographical portion of the aneurysm and the non-angiographical portion above it, that was the partial thrombus, were pulsating together. Considering that this was a rupture case and the aneurysm increased in a short period like $\mathrm{Xu}$ et al., ${ }^{1)}$ 

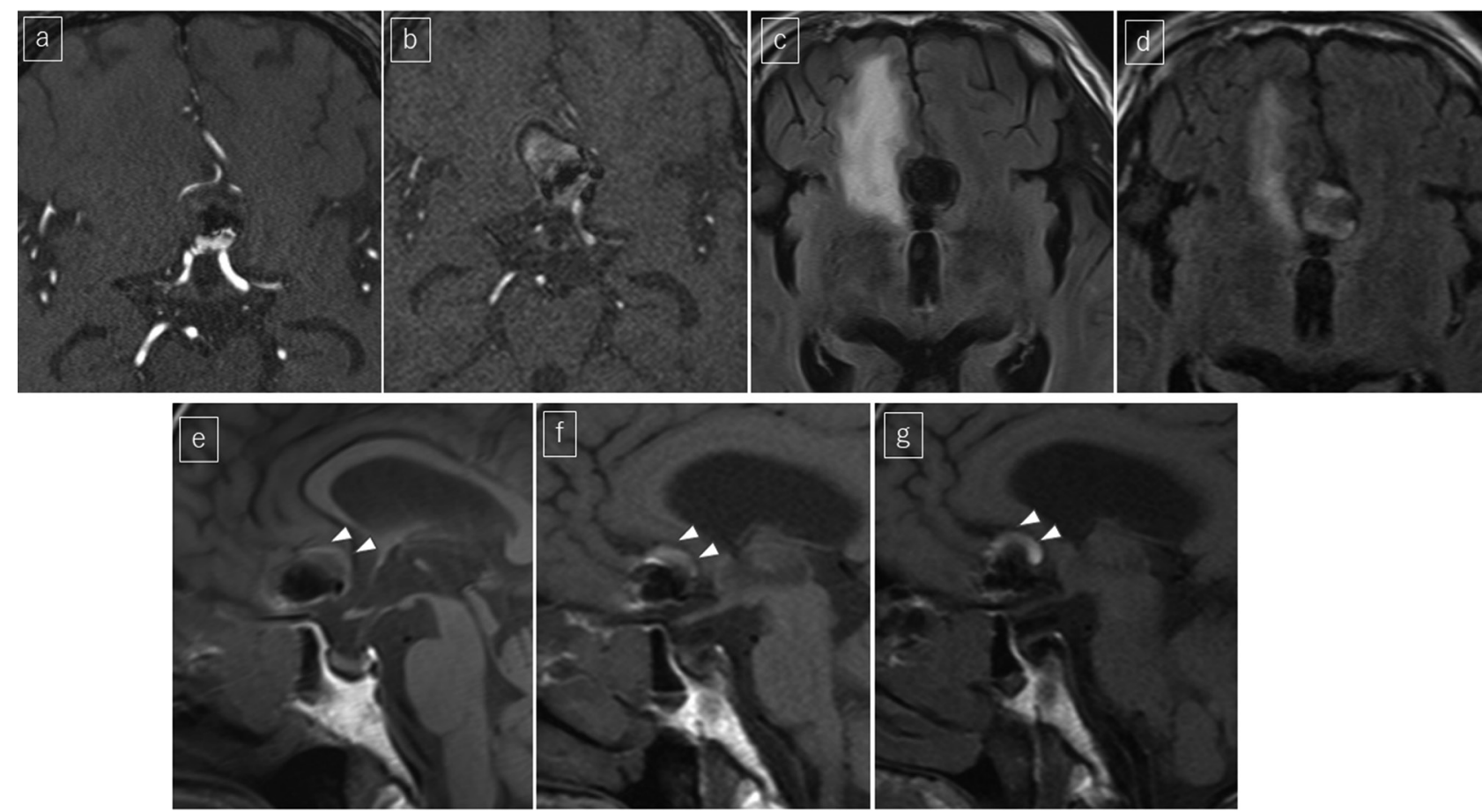

Fig. 3 Follow-up MRIs were performed several times after the first treatment. However, the neck remnant enlarged. (a) MRA immediately after first the treatment. (b) MRA 8 months after first treatment. The neck remnant part enlarged. (c) MRI FLAIR image immediately after the first treatment. (d) MRI FLAIR image 8 months after the first treatment. Cerebral edema around the aneurysm decreased. (e) T1-weighted image immediately after the first treatment. The presence of a partial thrombus of the aneurysmal upper neck segment was demonstrated. Arrow head: a partial thrombus segment. (f) T1-weighted image 3 months after the first treatment. Arrow head: a partial thrombus segment. (g) T1-weighted image 8 months after the first treatment. The intensity of the partial thrombus changed over time and the aneurysmal size increased. The enlarged aneurysm compressed the corpus callosum. Arrow head: a partial thrombus segment. FLAIR: fluid-attenuated inversion recovery, MRA: magnetic resonance angiography, MRI: magnetic resonance imaging.

we thought that the wall might have become thin and fragile. Therefore, we considered that the pulsation of the blood flow made the aneurysm wall appear to be pulsating and we could detect the pulsation of the aneurysm. Second, the entire aneurysmal wall was not pulsating. However, the portion of the partial thrombus extended and contracted repeatedly, and the angiographic portion of the aneurysm pulsated. Superficially, the aneurysmal wall did not seem to be pulsating. Considering the fact that the signals of the thrombus showed changes over time in the MRI images, there was a possibility that the thrombus was as fragile as the aneurysmal wall and the pulsation of the blood flow made the thrombus appear to be pulsating. We thought that the underlying vulnerability of the aneurysm wall was the reason we were able to visualize the aneurysm beat for both reasons. Then, it was expected that the wall was thin and fragile as in $\mathrm{Xu}$ et al case. ${ }^{1)}$ There was no significant increase in intracranial pressure in the images of our case. Therefore, Adel et al.'s beating mechanism was considered to be different from ours. ${ }^{2)}$ In our case, we considered that these were the only two possibilities as mentioned above.

As far as we have studied, no article has been found to mention whether cases of pulsating aneurysms were likely to rupture. However, Xu et al. reported that the direct finding of a pulsating and rapidly enlarging aneurysm was that the wall was thin and fragile. ${ }^{1)}$ Therefore, the same was expected in our case, so we decided to intervene urgently.

\section{Conclusion}

In this study, we present a case of pulsatile intracranial aneurysm with a peculiar angiographic appearance. The aneurysm's mechanism of pulsation 

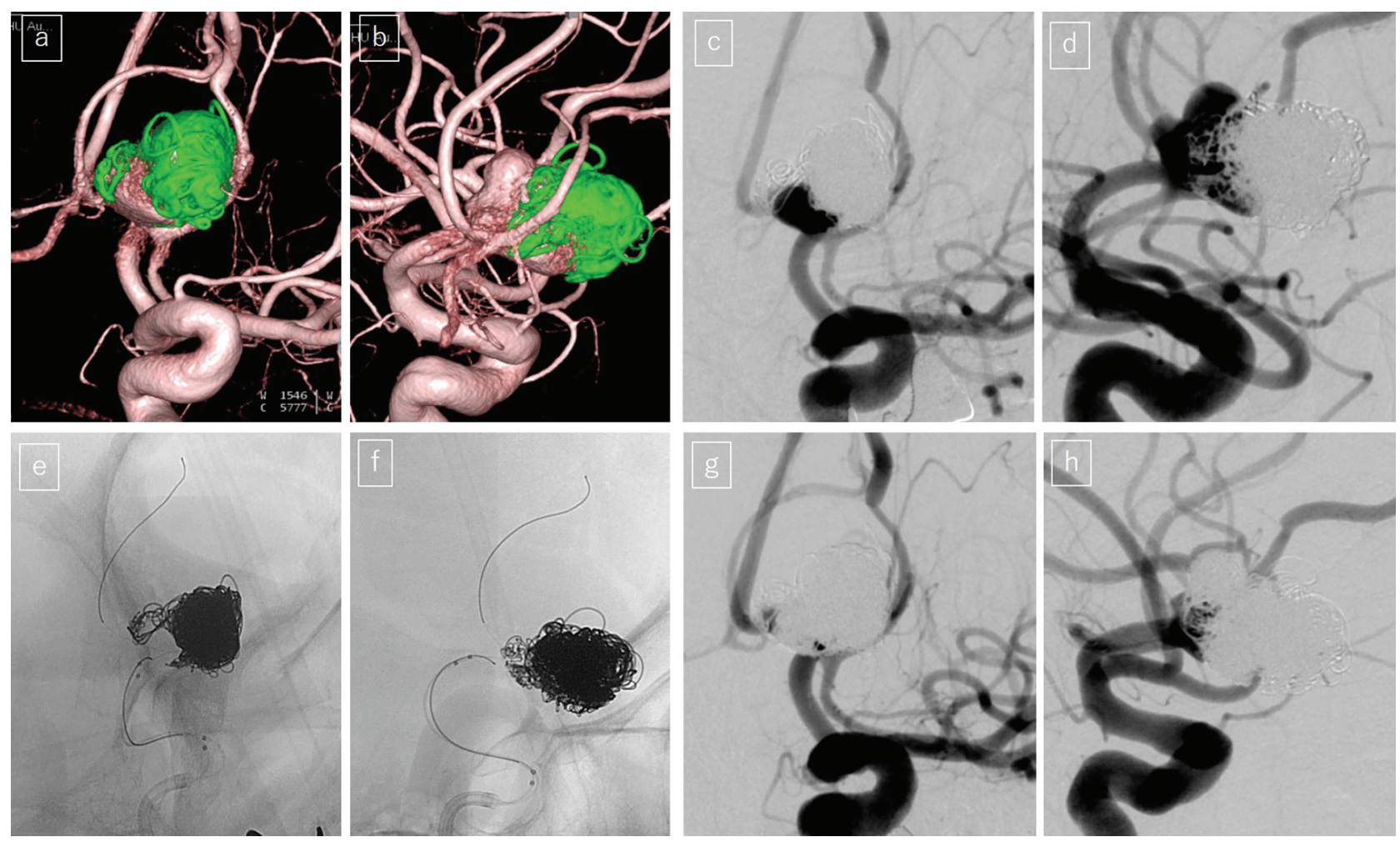

Fig. 4 We performed a re-treatment for the anterior communicating artery aneurysm with stent assist coil embolization. We conducted the procedure more carefully, detecting the pulsatile aneurysmal neck. (a and b) 3D-RA VR image. (c and d) This was the working angle, clearly demonstrating the aneurysm's neck part. We treated the patient with several working angles, as follows: an angle to demonstrate the distal anterior cerebral artery and one for the anterior communicating artery. (e and f) We advanced a micro-guidewire to the distal anterior cerebral artery. Then, we advanced a micro-catheter. ( $g$ and $h$ ) This was the final angiography of the working angle. A slight neck remnant was demonstrated. However, a perforator arising from the neck was to be preserved. 3D-RA: three-dimensional rotational angiography, VR: volume rendering.

was not validated. However, the wall of the pulsatile aneurysm might be thin and fragile. Thus, patients with such conditions must be treated cautiously.

\section{Conflicts of Interest Disclosure}

None.

Authors who are members of The Japan Neurosurgical Society (JNS) have registered online Selfreported COI Disclosure Statement Forms through the website for JNS members.

This summary statement ultimately will be published if the article is accepted.

\section{References}

1) Xu D, Xie X, You C: Angiographic appearance of pulsatile blister aneurysm. World Neurosurg 110: 371-372, 2018

2) Malek AM, Halbach VV, Holmes S, et al.: Beating aneurysm sign: angiographic evidence of ruptured aneurysm tamponade by intracranial hemorrhage. Case illustration. J Neurosurg 91: 517, 1999

Corresponding author: Takashi Izumi, MD, PhD Department of Neurosurgery, Nagoya University Graduate School of Medicine, 65 Tsurumaicho, Shouwa-ku, Nagoya, Aichi 466-8550, Japan. e-mail: my-yuzu@med.nagoya-u.ac.jp 